chen größtenteils auf Privatpersonen basierenden Bürger*innenenergiegesellschaften zu strategischen, von Unternehmen initiierten, vermeintlichen Bürger*innenenergiegesellschaften (vgl. Kapitel 5.2.8).

Insgesamt werden in den Debatten um die „Energiewende“ sowie erneuerbare Energien kooperative Vorstellungen des Zusammenwirkens marginalisiert und die Gestaltung der Energiepolitik marktwirtschaftlichen Prinzipien unterworfen. Machtasymmetrien im Wettbewerb werden bis auf wenige Ausnahmen (bspw. der Verweis auf die Macht der , großen Vier', vor allem durch SPD, GRÜNE und LINKE) nicht artikuliert. Es dominiert die implizite Annahme von gleichen Ausgangsbedingungen, eines Wettbewerbs unter Gleichen. Normative, sozial-ökologische Handlungsorientierungen geraten aus dem Blick. Dies führt zu einer diskursiven Verfestigung der gesellschaftlichen Naturverhältnisse, in denen die Natur der Wettbewerbslogik unterworfen wird (Krüger 2015: 101; Görg 2003: 140). ${ }^{16}$ Natur wird somit nicht als gleichberechtigte Akteurin in einem ökonomischen Kooperationsprozess gefasst, sondern wird nutzenorientiert angeeignet und als Mittel zum Zweck betrachtet. Arbeit wird nur im Sinne von Erwerbsarbeit adressiert, Sorgearbeit ist kein Gegenstand der Debatten. Kooperative, sozial-ökologische, geschweige denn (re)produktive politische Praktiken werden so verunmöglicht.

\title{
6.3 AM GUTEN LEBEN ORIENTIERTE „ENERGIEWENDE“?
}

In Kapitel 3.2.1 wurde Orientierung am für das gute Leben Notwendigen als handlungsleitendes Prinzip einer vorsorgenden Wirtschaftsweise eingeführt und zur Orientierung an monetären, rein marktorientierten Prinzipien abgegrenzt. Im Folgenden analysiere ich, welche handlungsleitenden Prinzipien sich in den untersuchten Debatten um Energiepolitik identifizieren lassen und welche Zielsetzungen und Wohlstandsvorstellungen damit verbunden werden.

Ein handlungsleitendes Prinzip des Narrativs der ,ökologischen Modernisierung‘ der 14. und 15. Legislaturperiode ist nicht unbedingtes Wachstum um jeden Preis, sondern ein Wachstum, welches mit ökologischen Zielvorstellungen vereinbar sein soll. Neben ökonomischen Zielen kommt auch der Erhalt der natürlichen Lebensgrundlagen als Lebensbedürfnis in den Blick. Reine Gewinnmaximierung wird nicht zur alleinigen Zielsetzung ökologischer Modernisierung erklärt: So werden überwiegend profitorientierte Motivationen wie bspw. der ,großen Vier‘ auch von der Regie-

ten sind im Vergleich zu den übrigen Bürgerenergiegesellschaften als eher nicht zentral gesteuert zu bezeichnen. Im Fall von GmbH und Co. KGs stehen meist Initiatoren in Form von Projektentwicklern, Flächeninhabern oder Ankerinvestoren bereit, die den Gründungsprozess der Bürgerenergiegesellschaft strukturieren. Im Gegensatz dazu sind in Energiegenossenschaften ehrenamtliche Gremienstrukturen anzutreffen, deren Motivation zu einem Großteil durch Altruismus erklärt werden muss“ (Kahla u.a. 2017: 25).

16 Auch wenn die oben dargestellten Effekte des EEG für verständige und vorsorgendverantwortlicher Kooperation außerhalb des Parlaments zum Teil andere gesellschaftliche Naturverhältnisse ermöglichen oder aufscheinen lassen (bspw. in Energiegenossenschaften, vgl. Amri-Henkel/Hofmeister (2018)). 
rungskoalition kritisiert. Diese würden die erneuerbaren Energien ablehnen, weil sich damit „kein Geld verdienen“ ließe (SPD 06.06.2008: 17731; vgl. Kapitel 5.2.10). Eine gemeinwohlorientierte im Gegensatz zu einer profitorientierten Ausrichtung wird aber nicht auf die Ökonomie als Ganzes übertragen. Es wird keine grundsätzliche Kritik an den ökonomischen Zielsetzungen und Voraussetzungen von Wirtschaftswachstum geübt, so wird Wachstum nicht direkt mit Ressourcenverbrauch oder Treibhausgasemissionen in Beziehung gesetzt. Vielmehr liegt die implizite Prämisse zu Grunde, dass Wachstum und Ökologie keine grundsätzlichen Gegensätze darstellen, sondern miteinander in Einklang zu bringen seien, wenn ein ,anderes', ökologischeres Wachstum angestrebt werde (vgl. auch Krüger 2015: 107) ${ }^{17}$ : So solle der nachhaltige „Erhalt der natürlichen Lebensgrundlagen“ gleichzeitig „Gerechtigkeit und Wachstum“ sichern (Koalitionsvertrag 2002: 7; vgl. Kapitel 5.2.1). Umgekehrt wird davon ausgegangen, dass Naturzerstörung dem Wirtschaftswachstum schade. Beidem liegt keine (re)produktive Perspektive im Sinne einer Vermittlung von Ökologie und Ökonomie zugrunde, sondern es dominieren, wie bereits gezeigt, marktökonomieorientierte Story-Lines. Im fantasmatischen Narrativ ,ökologischer Modernisierung' werden so Forderungen aus der Umweltbewegung mit der Forderung des Wirtschaftswachstums äquivalenziert, wodurch letztlich eine Voraussetzung für die Ausbreitung einer hegemonialen marktwirtschaftlichen Formation geschaffen wird. So beziehen sich bereits in der 14. und 15. Legislaturperiode CDU, CSU, GRÜNE und SPD - wenn auch in unterschiedlicher Weise - auf ein ökologischeres Wachstum, ${ }^{18}$ was auf die beginnende Vermischung der fantasmatischen Narrative ,ökologischer Modernisierung' und ,marktwirtschaftlicher Energiepolitik' in einem technokratisch-managementorientierten Ökokonses verweist. Dagegen fordert die PDS eine Abkehr vom Wachstumsmodell ${ }^{19}$ und kritisiert die Blindheit des Marktes ,gegenüber gesellschaftlichen Bedürfnissen“ (PDS 25.11.1999: 6654).

In der 16. Legislaturperiode wird die Wachstumsdynamik des Sektors erneuerbarer Energien wird mythenhaft aufgeladen (SPD 06.06.2008: 17730; vgl. Kapitel 5.2.3). Ökonomische Zielsetzungen werden dabei im Koalitionsvertrag mit den ,sozialen Anliegen der Menschen im Sinne des nachhaltigen Wirtschaftens" verbunden (Koalitionsvertrag 2005: 65). In den Debatten um die Energiepolitik spielen diese dann allerdings kaum mehr eine Rolle (vgl. auch Kapitel 5.1.2). Anstatt Energiepolitik an einer Orientierung an sozial-ökologischen Bedürfnissen oder gar am für das gute Leben Notwenigen auszurichten, geht es vielfach darum, international eine Vor-

17 Vgl. z.B.: „Unsere Politik der Energiewende bringt mehrfach Dividende: Sie ist gut für Umwelt und Wirtschaft und sie schafft Arbeitsplätze“ (SPD 27.06.2002: 24781).

18 Vgl. z.B.: „Eine ökonomische Grundwahrheit ist, dass nur technischer Fortschritt nachhaltiges Wachstum begründen kann“ (CDU 24.02.2005: 14980). „Mehr Wohlstand für alle ist nur durch nachhaltiges Wirtschaftswachstum erreichbar“ (CSU 07.11.2002: 464). „Der Emissionshandel soll Impulse für ein nachhaltiges Wachstum geben“ (GRÜNE 16.01. 2004: 7664).

19 Vgl. z.B.: „Ich denke, hier gibt es technisch-technologische Grenzen. Langfristig ist das Wachstumsmodell, welches auch die rot-grüne Koalition hochhält, eine Sackgasse“ (PDS 25.02.2000: 8436). 
reiterposition im Bereich erneuerbarer Energien einzunehmen ohne das eigene Wohlstandsmodell in Frage zu stellen (vgl. Kapitel 5.2.6).

Aus einer kritischen Positionierung heraus thematisiert die LINKE in der 16. Legislaturperiode die „Energiewende“ im Kontext von sozialen Bedürfnissen, wie Sozialstandards bei Arbeitsplätzen (LINKE 06.06.2008: 17736) oder Frieden (LINKE 02.07.2009: 25694).

In der 17. Legislaturperiode wird der Stellenwert von Wachstum als Zweck politischer Praktiken bereits durch den Titel des Koalitionsvertrags verdeutlicht: „Wachstum. Bildung. Zusammenhalt" (Koalitionsvertrag 2009). Dabei wird weiter an der Vorstellung ökologischen Wachstums festgehalten: So solle der Ressourcenverbrauch durch Energieeffizienz derart reduziert werden, dass der gleiche „Anteil am Bruttosozialprodukt mit nur der Hälfte der Energie produzier[t]“ werden kann (CDU 28.10.2010: 7167). Der Energiebereich diene der Sicherung einer „nachhaltige[n] wirtschaftliche Prosperität“ (17/3049: 2). Gleichzeitig treten ökologische Zielsetzungen von Wachstum in den Hintergrund: Das EEG wird von schwarz-gelb vor allem positiv gesehen, weil es ein „,massives Wachstum“ im Sektor erneuerbarer Energien verursacht habe und dadurch zum Treiber für „die Modernisierung der Energieinfrastruktur" wurde (17/3049: 4). Als Akteur*innen werden überwiegend private Unternehmen gesehen, weshalb deren wirtschaftliche Interessen Eingang in politische Zielsetzungen finden, während die (Lebens-)Bedürfnisse anderer Akteur*innen ausgeblendet werden (FDP 28.10.2010: 7173; vgl. Kapitel 5.2.4). Durch den Fokus auf marktwirtschaftliche und technologische Zielsetzungen kommen die Auswirkungen der Laufzeitverlängerung auf die Lebensbedingungen in den Reden der Regierungskoalition gar nicht erst in den Blick. Gleichzeitig kritisiert die Opposition die Verlängerung der Laufzeiten als rein profitorientiert (vgl. Kapitel 5.2.10).

Durch die Nuklearkatastrophe von „Fukushima“ gerät der Widerspruch der Kernenergie mit grundlegenden Lebensbedürfnissen in den Blick (z.B. Gesundheit, intakte Lebensgrundlagen, etc.). Mit der Artikulation von „Energiewende“ soll dieser Widerspruch überwunden werden. Sie wird implizit zum Leitprojekt für nachhaltiges Wachstum in einem Industrieland erhoben. Ziel sei es, die „Energiewende“ „ohne Wohlfahrtsverluste zu stemmen“ (FDP 13.06.2013: 31292) (vgl. Kapitel 5.2.6). Wachstum bleibt Ziel und Voraussetzung der „Energiewende ${ }^{20 ، ،}$ (vgl. Kapitel 5.4), während die Verbindung der Energiepolitik zu sozial-ökologischen Lebensbedürfnissen weiterhin aus den politischen Debatten externalisiert bleibt (vgl. z.B. CSU 13.06.2013: 31584; vgl. Kapitel 5.4). Wachstumsorientierte Zielsetzungen von „Energiewende“ werden dabei nicht nur von den Regierungsparteien CDU, CSU und FDP artikuliert, sondern teilweise auch von SPD und GRÜNEN. ${ }^{21}$

20 So machen laut FDP die „,erhöhten Wachstumszahlen“ „,den zugegeben riskanten Weg dieser Energiewende“ leistbar (FDP 30.06.2011: 13396).

21 Vgl. z.B.: „Wir sind mit der Energiewende angetreten, um zu zeigen [...], dass eine hochentwickelte Volkswirtschaft wie die deutsche es schafft, sich von nuklearer, langfristig aber auch von fossiler Energieversorgung zu befreien, ohne dabei ihren wirtschaftlichen Erfolg zu gefährden“ (SPD 27.06.3014: 3932). Vgl. z.B.: Deutschland steht „an der Schwelle, im 21. Jahrhundert Vorreiter für die Green Economy zu sein“ (GRÜNE 30.06.2011: 13382). 
Da eine Einbettung der „Energiewende“ in Grundfähigkeiten, bzw. sozial-ökologische Bedürfnisse kaum erfolgt, erscheint eine marktökonomische Ausrichtung insgesamt als Selbstzweck und übergeordnetes Ziel.

Die Vorstellung ,ökologischen Wachstums‘ wird durch die Ausbreitung der hegemonialen marktwirtschaftlichen Formation im Zusammenhang mit dem Narrativ des technokratisch-managementorientierten Ökokonsenses zwar gestärkt, andererseits treten ökologische Zielsetzungen eines solchen Wachstums immer weiter in den Hintergrund. So sind vor allem Kosten-Nutzen Kriterien in den Debatten um die „Energiewende“ der 18. Legislaturperiode zentral. Die Nennung ökologischer Zusammenhänge der „Energiewende“ bleibt auf wenige Ausnahmen beschränkt (bspw. die Vermeidung von „Nutzungskonkurrenzen mit dem Arten- und Naturschutz“ bei der Bioenergie (Koalitionsvertrag 2013: 54; Kapitel 5.1.2). Eine „Energiewende“ erscheint nur so lange legitim, als sie ihren ökonomischen Zweck nicht gefährdet. ${ }^{22}$

Insgesamt lässt sich schließen, dass mit Aufkommen des Narrativs der ,ökologischen Modernisierung' neben ökonomischen Zielsetzungen ökologische Zwecke des Wirtschaftens zwar Einzug in den Diskurs erhalten. Allerdings wird Ökonomie auch hierbei keine rein (lebens)dienende Funktion zugeschrieben, sondern sie wird immer gleichzeitig selbst als Zweck betrachtet. Die Frage, wie Aushandlungsräume für eine am guten Leben orientierte Energiepolitik geschaffen werden können, ist kein politisches Thema. Im Gegenteil, die Notwendigkeit dafür kommt gar nicht erst in den Blick, weil das auf monetärer Wertmessung beruhende Wohlstandsmodell und das daraus abgeleitete Ziel des Wirtschaftswachstums unhinterfragt bleiben. Die Frage der Lebensdienlichkeit der Energiepolitik scheint mit „Fukushima“ zwar kurzzeitig auf, erscheint aber mit der „Energiewende“ vermeintlich beantwortet. Obwohl die „Energiewende“ mindestens implizit mit der Sinn- und Legitimationsfrage von Wirtschaften in Verbindung steht (z.B. Erhalt von natürlichen Lebensgrundlagen, Generationengerechtigkeit) und dafür auch unabhängig der vorliegenden Ergebnisse Potenziale bietet, werden diese Fragen in den Debatten nach „Fukushima“ permanent aus dem Politischen gedrängt und durch marktökonomische Zielsetzungen ersetzt. Die „Energiewende“ als soll als Teil der glücksverheißenden Dimension des technokratisch-managementorientierten Ökokonsens der Marktökonomie zum Erfolg verhelfen (oder diese mindestens nicht behindern). Die Frage nach dem Wozu der „Energiewende“ wird in den Debatten eher mit Wachstum, Effizienz oder der internationalen Reputation Deutschlands beantwortet, als mit dem guten Leben - quantitativer Wohlstand, bemessen an Wirtschaftswachstum, erscheint als Effekt einer marktorientierten „Energiewende“. Durch den Fokus auf am Markt geäußerte Präferenzen, kommen vermeintlich private Bedürfnisse oder die Versorgungswirtschaft nicht in den Blick.

22 Vgl. z.B.: Es gehe ,nicht nur um den Ausbau der erneuerbaren Energien auf Teufel komm raus, sondern auch darum, [...] vernünftige wirtschaftliche Strukturen“ zu erhalten (SPD 01.06.2016: 16978). 